\title{
Facile Synthesis of Pd-Ir Nanocubes for Biosensing
}

\author{
Jiuxing Li and Yingfu Li* \\ Department of Biochemistry and Biomedical Sciences, Michael G. DeGroote Institute of Infectious Disease Research (IIDR), \\ McMaster University, Hamilton, ON, Canada
}

Displaying extremely high peroxidase-like activity and uniform cubic structure enclosed by (100) facets, Pd-Ir nanocubes are an attractive nanomaterial for bioanalysis. However, there exists a great challenge to deposit atomic layers of Ir on the surface of Pd nanocubes due to the relatively low energy barrier of homogeneous nucleation of Ir atoms compared to heterogeneous nucleation. Here, a simple and surfactant-free approach is presented to synthesize Pd-Ir nanocubes with atomic Ir shell thickness in an aqueous solution at room temperature. Biomolecules such as antibodies and nucleic acids have free access to the surface of $\mathrm{Pd}-\mathrm{Ir}$ nanocubes. Applications of $\mathrm{Pd}-\mathrm{Ir}$ nanocubes in immunoassays and aptamer-based biosensors are realized, exploiting the excellent peroxidase activity and fluorescence quenching ability of Pd-Ir nanocubes. This work makes a significant step forward towards the practical utility of Pd-Ir nanocubes in bioanalysis.

\section{OPEN ACCESS}

Edited by:

Paul E.D. Soto Rodriguez,

Commissariat à l'Energie Atomique et aux Energies Alternatives (CEA),

France

Reviewed by: Ilaria Palchetti, University of Florence, Italy Dechao Chen,

Griffith University, Australia

*Correspondence: Yingfu $L i$ liying@mcmaster.ca

Specialty section: This article was submitted to Nanoscience,

a section of the journal Frontiers in Chemistry

Received: 13 September 2021 Accepted: 28 October 2021 Published: 24 November 2021

Citation:

Li J and Li Y (2021) Facile Synthesis of Pd-Ir Nanocubes for Biosensing. Front. Chem. 9:775220. doi: $10.3389 /$ fchem.2021.775220
Keywords: Pd-Ir nanocubes, peroxidase-mimicking nanozymes, immunoassay, aptamer, fluorescent colorimetric biosensors, PSA, RNase HII

\section{INTRODUCTION}

In recent years, nanomaterials have attracted increasing research interests in bioanalysis due to their outstanding optical, electronic, and catalytic properties (Link and El-Sayed, 1999; Burda et al., 2005; Willets and Van Duyne, 2007; Anker et al., 2008; Saha et al., 2012; Wei and Wang, 2013; Lin et al., 2014; Wu et al., 2019). Nanomaterials have been widely explored to achieve enzyme-like catalysis, electrocatalysis, specific adsorption of nucleic acids, efficient fluorescence quenching, creation of nanostructured electrodes, among other bioanalytical applications (Lu et al., 2009; He S. et al., 2010; Loh et al., 2010; Wang et al., 2010; Wang et al., 2011; Wei and Wang, 2013; Zhu et al., 2013; Lin et al., 2014; Tan et al., 2015; Peng et al., 2017; Wu et al., 2019). Peroxidase-mimicking nanozymes including metal oxides $\left[\mathrm{Fe}_{3} \mathrm{O}_{4}\right.$ (Gao et al., 2007; Wei and Wang, 2008), $\mathrm{Co}_{3} \mathrm{O}_{4}$ (Mu et al., 2012), $\mathrm{V}_{2} \mathrm{O}_{5}$ (André et al., 2011), and $\mathrm{CuO}$ (Chen et al., 2011)], carbon materials (Song et al., 2010a; Song et al., 2010b), and noble metal nanomaterials (Jia et al., 2002; He W. et al., 2010; Jv et al., 2010; He et al., 2011; Xia et al., 2014), have been employed as alternatives to horseradish peroxidase (HRP) for antibody conjugation and signal output in immunoassays, due to their easy synthesis and increased stability (Wei and Wang, 2013; Wu et al., 2019). However, most nanozymes are subject to shortcomings in size and morphology control, large batch-to-batch variation, and limited enhancement in catalytic activity. As for adsorption-based fluorescent bioanalysis, two-dimensional (2D) nanomaterials, such as graphene oxide (Lu et al., 2009), $\mathrm{MoS}_{2}$ (Zhu et al., 2013), $\mathrm{Ta}_{2} \mathrm{NiS}_{5}$ (Tan et al., 2015), and covalent organic framework (Peng et al., 2017) are representative nanomaterials, attributable to their fairly flat surfaces. However, these 2D nanomaterials also face problems of large batch-to-batch variations and varying performance.

Pd-Ir nanocubes have great potential to circumvent the issues encountered by nanomaterials in bioanalysis, due to their superior properties including extremely high catalytic activity, excellent 
stability, good batch consistency, efficient fluorescence quenching, and uniform cubic structure with flat (100) facets (Xia et al., 2014; Xia et al., 2015; Ye et al., 2017; Zhu et al., 2019a; Zhu et al., 2019b). However, it has been a great challenge to deposit atomic Ir layer on Pd nanocube surfaces, due to the relatively low energy barrier for homogeneous nucleation of Ir atoms compared to heterogeneous nucleation on Pd nanocubes (Stowell and Korgel, 2005; Migowski et al., 2010; Xia et al., 2014). Most synthesis reactions result in amorphous Ir-coated Pd nanocubes, which compromise their stability, synthesis reproducibility, and uniform structure (Liu et al., 2013; Zanata et al., 2019). Harsh conditions including high temperature, surfactants, organic solvent, and flow injection in an oil bath, are required to successfully deposit thin Ir layers on Pd nanocube surfaces (Xia et al., 2015; Ye et al., 2017; Zhu et al., 2019a; Zhu et al., 2019b). However, synthesis under such conditions are not compatible with biomolecular adsorption, not easy to scale up, not environmentally friendly, restricting various applications of Pd-Ir nanocubes in bioanalysis.

The synthesis of Pd-Ir nanocubes includes two steps: Pd nanocube synthesis and Ir deposition. Previously, we reported a simple one-pot method for Pd nanocube synthesis ( $\mathrm{Li}$ and $\mathrm{Li}$, 2021). Herein, we describe a simple and surfactant-free method for depositing atomic Ir shells on Pd nanocubes in an aqueous solution at room temperature. The Pd-Ir nanocubes show high peroxidase activity and free accessibility for biomolecules. The nanomaterials are further exploited for the development of an immunoassay to detect PSA (prostate-specific antigen) as well as fluorescent and colorimetric biosensors of $C$. difficile RNase HII.

\section{EXPERIMENTAL SECTION}

\section{Chemicals and Reagents}

Fluorescein-labelled DNA aptamer (FAM-ARH1t6: TTA CGT CAA GGT GTC ACT CCG CCA GGT GTG CGA CGG TCG T-FAM) were purchased from Integrated DNA Technologies (Iowa, United States) and purified by $10 \%$ denaturing PAGE (polyacrylamide gel electrophoresis). Sodium tetrachloropalladate (II) $\left(\mathrm{Na}_{2} \mathrm{PdCl}_{4}, 98 \%\right), \mathrm{L}$-ascorbic acid (AA, $\geq 99 \%$ ), polyvinylpyrrolidone (PVP, M.W. $\approx 55,000$ ), potassium bromide ( $\mathrm{KBr}, \geq 99 \%$ ), sodium hexachloroiridate (III) hydrate $\left(\mathrm{Na}_{3} \mathrm{IrCl}_{6} \bullet \mathrm{xH}_{2} \mathrm{O}, \mathrm{M} . \mathrm{W} .=473.9\right)$, sodium borohydride $\left(\mathrm{NaBH}_{4}\right.$, $98 \%$ ), sodium acetate ( $\mathrm{NaOAc}, \geq 99 \%$ ), acetic acid (HOAc, $\geq 99.7 \%$ ), hydrogen peroxide solution $\left(30 \% \mathrm{w} / \mathrm{v}\right.$ in $\left.\mathrm{H}_{2} \mathrm{O}\right)$, $3,3^{\prime}, 5,5^{\prime}$-tetramethylbenzidine (TMB, $\quad>99 \%$ ), dimethylformamide (DMF), sulfuric acid $\left(\mathrm{H}_{2} \mathrm{SO}_{4}, 95-98 \%\right)$, sodium phosphate dibasic $\left(\mathrm{Na}_{2} \mathrm{HPO}_{4}, \geq 99 \%\right)$, potassium phosphate monobasic $\left(\mathrm{KH}_{2} \mathrm{PO}_{4}, \geq 99 \%\right)$, sodium chloride $(\mathrm{NaCl}, \geq 99.5 \%)$, potassium chloride ( $\mathrm{KCl}, \geq 99 \%)$, hydrochloric acid ( $\mathrm{HCl}, 37 \%)$, horse radish peroxidase (HRP, $\geq 99 \%$ ), human prostate specific antigen (PSA, $\geq 99 \%$ ), bovine serum albumin (BSA, $\geq 98 \%$ ), and Tween-20 were all obtained from Sigma-Aldrich. HRP-goat anti-mouse IgG conjugate and goat anti-mouse IgG were from Thermo Fisher Scientific, Inc. Rabbit anti-PSA polyclonal antibody (pAb) and mouse anti-PSA monoclonal antibody (mAb) were both obtained from Abcam plc. C. difficile RNase HII is expressed and purified in our lab using E. coli cells containing an expression vector for the target protein.

\section{Synthesis of Pd-Ir Nanocubes}

Pd nanocubes with different sizes (7, 18 and $51 \mathrm{~nm})$ were prepared according to our previously reported method ( $\mathrm{Li}$ and Li, 2021). Pd-Ir nanocubes were synthesized by deposition of Ir atoms on the surface of Pd nanocubes. Briefly, Pd nanocubes $(50 \mu \mathrm{l}, 44 \mathrm{nM}, 18 \mathrm{~nm})$ were diluted by water $(1 \mathrm{ml})$ to $2.2 \mathrm{nM}$ in a centrifuge tube $(1.5 \mathrm{ml})$ and centrifuged at $14,000 \mathrm{rpm}$ for $10 \mathrm{~min}$. After removing the supernatant, the $\mathrm{Pd}$ nanocubes pellets were resuspended by water $(1 \mathrm{ml})$. Then, $\mathrm{Na}_{3} \mathrm{IrCl}_{6}(20 \mu \mathrm{l}, 10 \mathrm{mM})$ and $\mathrm{NaBH}_{4}(20 \mu \mathrm{l}, 100 \mathrm{mM})$ were added to the Pd nanocube solution sequentially and kept at room temperature for $1 \mathrm{~h}$. The $\mathrm{NaBH}_{4}$ $(20 \mu \mathrm{l}, 100 \mathrm{mM})$ addition process was repeated once, and the mixture was allowed to react at room temperature for $4 \mathrm{~h}$. Pd-Ir nanocubes were obtained and stored at room temperature before use. The concentration of Pd-Ir cubes synthesized by this method was $2.2 \mathrm{nM}$ according to the concentration of $\mathrm{Pd}$ nanocubes.

\section{Steady-State Kinetic Assays of Peroxidase-like Activity of Pd-Ir Nanocubes} The peroxidase-like activity of $\mathrm{Pd}-\mathrm{Ir}$ nanoparticles was demonstrated by catalyzing the oxidation of TMB by $\mathrm{H}_{2} \mathrm{O}_{2}$. Pd-Ir nanocubes $\left(50 \mu \mathrm{l}, 1.376 \times 10^{-13} \mathrm{M}\right)$ were mixed with TMB substrate $\left(50 \mu \mathrm{l}\right.$, containing $1.6 \mathrm{mM} \mathrm{TMB}, 4 \mathrm{M} \mathrm{H}_{2} \mathrm{O}_{2}$, $40 \mathrm{mM}$ acetate buffer, $\mathrm{pH}=4.0$ ) in the wells of a 96-well microtiter plate. After reacting at room temperature for $20 \mathrm{~min}$, the reaction was terminated by $\mathrm{H}_{2} \mathrm{SO}_{4}(20 \mu \mathrm{l}, 2 \mathrm{M})$. A microplate reader was used to measure the absorbance of the TMB oxidized product at $450 \mathrm{~nm}$.

A steady-state kinetic assay of Pd-Ir nanocubes for catalyzing the oxidation of TMB by $\mathrm{H}_{2} \mathrm{O}_{2}$ was conducted by monitoring the catalytic reaction in a real-time manner. Pd-Ir nanocubes $(50 \mu \mathrm{l}$, $\left.2.752 \times 10^{-13} \mathrm{M}\right), \mathrm{H}_{2} \mathrm{O}_{2}(50 \mu \mathrm{l}, 8 \mathrm{M})$, and TMB substrate $(100 \mu \mathrm{l}$, $1.6 \mathrm{mM}$ TMB in $40 \mathrm{mM}$ acetate buffer, $\mathrm{pH}=4.0$ ) were mixed in a cuvette with a path length of $1 \mathrm{~cm}$. The absorbance at $653 \mathrm{~nm}$ was measured by UV-Vis absorption spectroscopy for $300 \mathrm{~s}$ with intervals of $12 \mathrm{~s}$. According to the extinction coefficient of TMB oxidized product at $653 \mathrm{~nm}\left(3.9 \times 10^{-4} \mathrm{M}^{-1} \bullet \mathrm{cm}^{-1}\right)$, the initial reaction velocity $(v)$ at a specific concentration of substrate was calculated by the initial slope of UV-Vis absorption kinetic curves. The apparent kinetic parameters could be calculated by the Michaelis-Menten equation $v=V_{\max } \times(S) /\left[K_{m}+(S)\right]$, where $v$ is the initial reaction velocity, $\mathrm{V}_{\max }$ is the maximal reaction velocity, (S) represents the concentration of substrate, and $K_{m}$ represents the Michaelis constant.

\section{Functionalization of Pd-Ir Nanocubes With Antibodies}

Pd-Ir nanocubes are functionalized with goat anti-mouse IgG by physical adsorption. Briefly, goat anti-mouse $\operatorname{IgG}(5 \mu \mathrm{l}, 1 \mathrm{mg} / \mathrm{ml})$ and mPEG-SH $(10 \mu \mathrm{l}, 100 \mu \mathrm{M})$ were added to Pd-Ir nanocubes 
( $1 \mathrm{ml}, 0.55 \mathrm{nM}$ ) followed by incubation at room temperature for $1 \mathrm{~h}$. Then, BSA $(200 \mu \mathrm{l}, 10 \% \mathrm{w} / \mathrm{v})$ was introduced to the mixture to block Pd-Ir nanocubes by incubation at room temperature for $1 \mathrm{~h}$. Finally, goat anti-mouse IgG-conjugated Pd-Ir nanocubes were washed twice with BSA solution $(1 \mathrm{ml}, 1 \% \mathrm{w} / \mathrm{v})$ by centrifuging at $10,000 \mathrm{rpm}$ for $5 \mathrm{~min}$. Finally, the goat antimouse IgG-conjugated Pd-Ir nanocubes were resuspended in storage buffer $\left(1 \mathrm{ml}, 10 \mathrm{mM} \mathrm{Na} \mathrm{NPO}_{4}, 1.8 \mathrm{mM} \mathrm{KH}_{2} \mathrm{PO}_{4}\right.$, $137 \mathrm{mM} \mathrm{NaCl}, 2.7 \mathrm{mM} \mathrm{KCl}, 0.05 \% \mathrm{v} / \mathrm{v}$ Tween-20, 10\% w/v BSA) and kept at $4^{\circ} \mathrm{C}$ before use.

\section{Pd-Ir Nanocubes for Prostate-Specific Antigen Immunoassay}

Rabbit anti-PSA pAb $(100 \mu \mathrm{l}, 5 \mu \mathrm{g} / \mathrm{ml}$, in PBS $)$ was first attached to the wells of a microtiter plate by incubation at $4^{\circ} \mathrm{C}$ for $12 \mathrm{~h}$, followed by three washes with PBST $(200 \mu \mathrm{l}$, $10 \mathrm{mM} \mathrm{Na}_{2} \mathrm{HPO}_{4}, 1.8 \mathrm{mM} \mathrm{KH}_{2} \mathrm{PO}_{4}, 137 \mathrm{mM} \mathrm{NaCl}, 2.7 \mathrm{mM}$ $\mathrm{KCl}, 0.05 \% \mathrm{v} / \mathrm{v}$ Tween-20). Then, the wells were blocked with blocking buffer $\left(200 \mu \mathrm{l}, 10 \mathrm{mM} \mathrm{Na} \mathrm{HPO}_{4}, 1.8 \mathrm{mM} \mathrm{KH} \mathrm{PO}_{4}\right.$, $137 \mathrm{mM} \mathrm{NaCl}, 2.7 \mathrm{mM} \mathrm{KCl}, 0.05 \% \mathrm{v} / \mathrm{v}$ Tween-20, 2\% w/v BSA) by incubation at $37^{\circ} \mathrm{C}$ for $1 \mathrm{~h}$, followed by washing for three times. Afterwards, PSA-containing samples in antibody

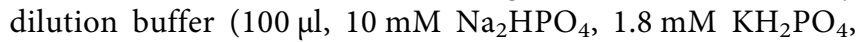
$137 \mathrm{mM} \mathrm{NaCl}, 2.7 \mathrm{mM} \mathrm{KCl}, 0.05 \% \mathrm{v} / \mathrm{v}$ Tween-20, 2\% w/v BSA) were added and shaken at $100 \mathrm{rpm}$ at $25^{\circ} \mathrm{C}$ for $2 \mathrm{~h}$. After removing the samples, the wells of the microtiter plate were washed three times again with PBST. Next, mouse anti-PSA $\mathrm{mAb}(100 \mu \mathrm{l}, 2 \mu \mathrm{g} / \mathrm{ml}$ in antibody dilution buffer $)$ was introduced to associate with PSA by shaking at $100 \mathrm{rpm}$ at $25^{\circ} \mathrm{C}$ for $1 \mathrm{~h}$. After washing the wells three times, goat antimouse IgG conjugated Pd-Ir nanocubes $(100 \mu \mathrm{l}, 0.138 \mathrm{nM})$ were added to the wells followed by shaking at $100 \mathrm{rpm}$ at $25^{\circ} \mathrm{C}$ for $1 \mathrm{~h}$. Pd-Ir nanocubes were removed by washing three times using PBST. Finally, TMB substrate $(100 \mu \mathrm{l}, 0.8 \mathrm{mM}$ TMB, $2 \mathrm{M}$ $\mathrm{H}_{2} \mathrm{O}_{2}, 20 \mathrm{mM} \mathrm{NaOAc} / \mathrm{HOAc}, \mathrm{pH}=4.0$ ) was added to initiate the catalytic reaction by incubation at $25^{\circ} \mathrm{C}$ for $20 \mathrm{~min}$. The reaction was terminated with $\mathrm{H}_{2} \mathrm{SO}_{4}(20 \mu \mathrm{l}, 2 \mathrm{M})$. UV-Vis absorbance at $450 \mathrm{~nm}$ was measured and compared to a standard working curve to determine the concentration of PSA.

In comparison, HRP-goat anti-mouse IgG conjugates were used to carry out the standard ELISA. All the experimental procedures of standard ELISA are the same as that of Pd-Ir nanocube-based immunoassay except the replacement of goat anti-mouse IgG-conjugated Pd-Ir nanocubes with HRP-goat anti-mouse IgG conjugates $(100 \mu \mathrm{l}, 0.5 \mu \mathrm{g} / \mathrm{ml})$ and the reduction of $\mathrm{H}_{2} \mathrm{O}_{2}$ concentration in TMB substrates to $10 \mathrm{mM}$.

\section{Pd-Ir Nanocube-Based Biosensor for $C$. Difficile RNase HII Detection}

All fluorescence spectra were measured by a Tecan microplate reader. In a typical assay, FAM-labelled DNA aptamer (FAMARH1t6, $10 \mu \mathrm{l}, 1 \mu \mathrm{M})$ was incubated with different concentrations of protein (0.5-64 nM) in HEPES buffer $(100 \mu \mathrm{l})$ at room temperature for $30 \mathrm{~min}$. Then, Pd-Ir nanoparticles $(100 \mu \mathrm{l}, 22 \mathrm{nM})$ were added and incubated at room temperature for $30 \mathrm{~min}$, followed by fluorescence measurements (excitation: $494 \mathrm{~nm}$; emission: $522 \mathrm{~nm}$ ).

For colorimetric detection of protein, FAM-labelled DNA aptamer, protein, and Pd-Ir nanoparticles were incubated as described above. Then, Pd-Ir nanocubes were diluted by water to a final concentration of $1.376 \times 10^{-13} \mathrm{M}$. Pd-Ir nanocubes $\left(50 \mu \mathrm{l}, 1.376 \times 10^{-13} \mathrm{M}\right)$ was mixed with TMB substrate $(50 \mu \mathrm{l}$, containing $1.6 \mathrm{mM} \mathrm{TMB}, 4 \mathrm{M} \mathrm{H}_{2} \mathrm{O}_{2}, 40 \mathrm{mM}$ acetate buffer, $\mathrm{pH}=$ 4.0) in the wells of a 96-well microtiter plate. After reacting at room temperature for $20 \mathrm{~min}$, the reaction was terminated by $\mathrm{H}_{2} \mathrm{SO}_{4}(20 \mu \mathrm{l}, 2 \mathrm{M})$. A microplate reader was used to measure the absorbance of the TMB oxidized product at $450 \mathrm{~nm}$.

\section{Characterizations}

The UV-Vis kinetic assays were conducted by UV-Vis absorption spectroscopy (Agilent Cary 60). The UV-Vis absorption spectra and fluorescence spectra were measured by a Tecan microplate reader. SEM analyses were performed on Hitachi S-4700 FE-SEM at $20 \mathrm{kV}$. TEM images were obtained using a JEOL transmission electron microscope (JEM-2010) operating at $200 \mathrm{kV}$. HRTEM images and EDX spectra were obtained on an FEI Titan 80-300 LB microscope operating at $300 \mathrm{kV}$. High angle annular darkfield (HAADF)-STEM analyses and EDX mapping were carried out on an aberration-corrected FEI Titan 80-300 HB Cubed microscope operating at $200 \mathrm{kV}$. The $\mathrm{pH}$ values of different buffer solutions were measured on a benchtop $\mathrm{pH}$ meter (Oakton $\mathrm{pH} 700)$.

\section{RESULTS AND DISCUSSION}

\section{Synthesis and Characterization of Pd-Ir Nanocubes}

The scheme to synthesize Pd-Ir nanocubes is shown in Figure 1A. Pd nanocubes were prepared according to our previously published method ( $\mathrm{Li}$ and $\mathrm{Li}, 2021$ ). Then, Ir is deposited directly on $\mathrm{Pd}$ nanocubes through the reduction of $\mathrm{Na}_{3} \mathrm{IrCl}_{6}$ by $\mathrm{NaBH}_{4}$. Because both Pd and Ir are ideal catalysts towards the hydrolysis of $\mathrm{NaBH}_{4}$ for $\mathrm{H}_{2}$ generation (Kaufman and Sen, 1985; Muir and Yao, 2011; Retnamma et al., 2011), two mechanisms are involved in the deposition of Ir on Pd nanocubes. First, $\mathrm{Pd}$ nanocubes catalyze the hydrolysis of $\mathrm{NaBH}_{4}$ to form hydrogen-capped $\mathrm{Pd}$ nanocubes, which reduce $\mathrm{IrCl}_{6}{ }^{3-}$ to form $\mathrm{Pd}-\mathrm{Ir}$ nanocubes. Second, $\mathrm{BH}_{4}{ }^{-}$acts as both reducing agent and capping agent to convert $\mathrm{IrCl}_{6}{ }^{3-}$ into hydrogen-conjugated $\mathrm{Ir}$, which deposits on $\mathrm{Pd}$ nanocubes with the generation of $\mathrm{H}_{2}$. This approach avoids the high temperature needed to assist fast diffusion of Ir (Xia et al., 2014), achieving surfactant-free PdIr nanocubes.

Pd nanocubes were characterized by scanning electron microscopy (SEM, Supplementary Figure S1A) and transmission electron microscopy (TEM, Supplementary Figure S1B). A product yield higher than $98 \%$ is achieved for $\mathrm{Pd}$ nanocubes with a size distribution of $18.2 \pm 1.8 \mathrm{~nm}$ (Supplementary Figure S1C). The typical high-resolution TEM (HRTEM) image of an individual Pd nanocube along 

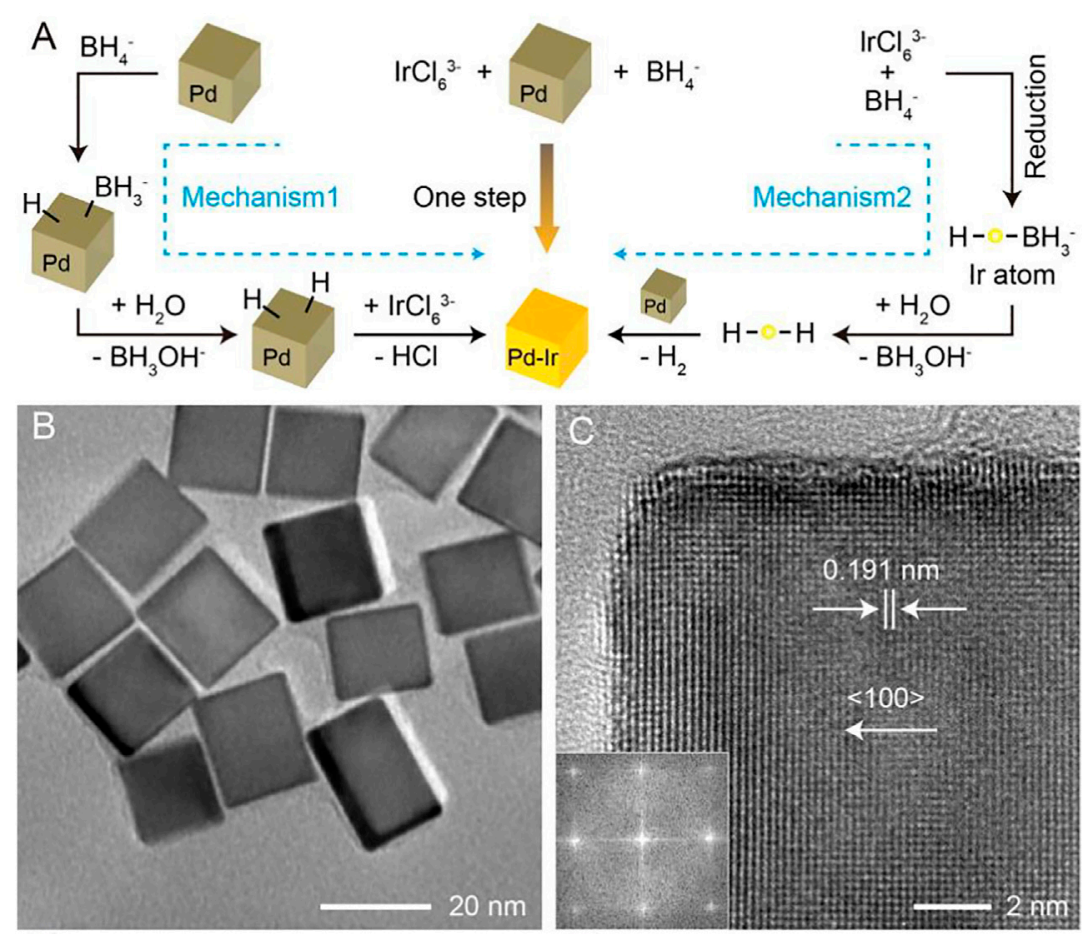

D

$\mathrm{E}$
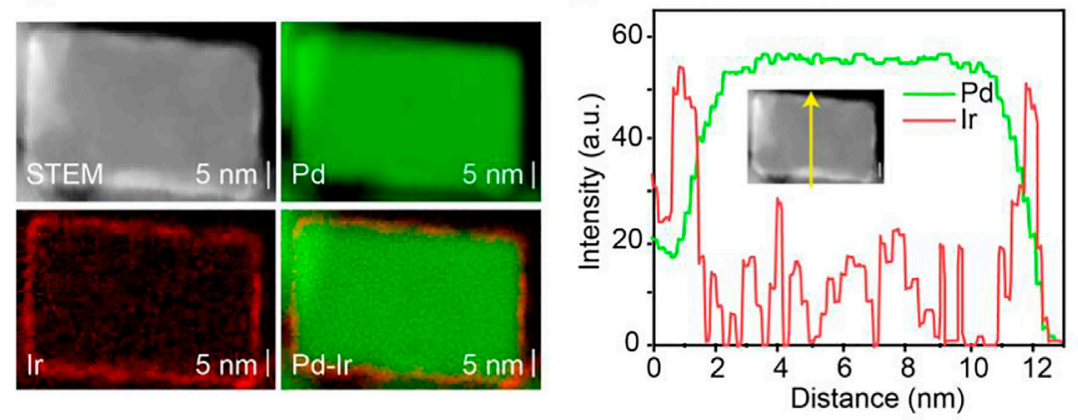

FIGURE 1 | (A) Scheme showing the mechanism of synthesis of Pd-Ir nanocubes. (B) The TEM image of Pd-Ir nanocubes. (C) The HRTEM image (inset: corresponding Fourier transform pattern) of a corner region of a Pd-Ir nanocube. (D) The HAADF-STEM image and EDX maps of a Pd-Ir nanocube. (E) Linear EDX analysis of a Pd-Ir nanocube.

the (100) zone axis clearly shows the (200) facets of face-centered cubic Pd, confirmed by the lattice spacing of $0.191 \mathrm{~nm}$ (Supplementary Figure S1D) (Lim et al., 2009; Xia et al., 2014; Xia et al., 2015; Zhu et al., 2019b). A thin Ir layer was deposited on the surface of Pd nanocubes by the reduction of $\mathrm{Na}_{3} \mathrm{IrCl}_{6}$ with $\mathrm{NaBH}_{4}$, achieving a smooth Ir surface (Figure 1B). The HRTEM image of a Pd-Ir nanocube (Figure 1C) along the (100) zone axis reveals continuous fringes, while the corresponding Fourier transform pattern (Figure 1C, inset) shows a square symmetry of the spots, indicating an epitaxial relationship between the single-crystal cubic $\mathrm{Pd}$ cores and $\mathrm{Ir}$ shells.

Owing to the difference of atomic number between Pd and Ir, thin Ir shells on the surface of Pd nanocubes can be resolved in the high-angle annular dark-field scanning transmission electron microscopy (HAADF-STEM) image (Supplementary Figure
S1E). Statistical analysis of Pd-Ir nanocubes (Supplementary Figure S1F) demonstrates an average edge length of $19.8 \pm$ $3.1 \mathrm{~nm}$, which is $1.6 \mathrm{~nm}$ greater than that of $\mathrm{Pd}$ nanocubes, therefore signifying an average thickness of $0.8 \mathrm{~nm}$ Ir shells (about $3 \mathrm{Ir}$ atoms) on each face of Pd nanocubes. In addition, the presence of Ir on $\mathrm{Pd}$ nanocubes was further verified by energy-dispersive X-ray spectroscopy (EDX) analysis (Supplementary Figure S1G), and the distribution of Ir on Pd nanocube surfaces was visualized by EDX elemental mapping (Figure 1D). The HAADF-STEM image (Figure 1D) of an individual $\mathrm{Pd}$-Ir nanocube shows a cubic structure surrounded by a brighter shell, which is ascribed to the higher atomic number of Ir compared to Pd. Pd and Ir are highlighted green and red respectively. The particle core is dominated by $\mathrm{Pd}$ while the shell is mainly composed of a uniform Ir layer. Linear EDX scan analysis (Figure 1E) confirms the Ir shell having a thickness of 

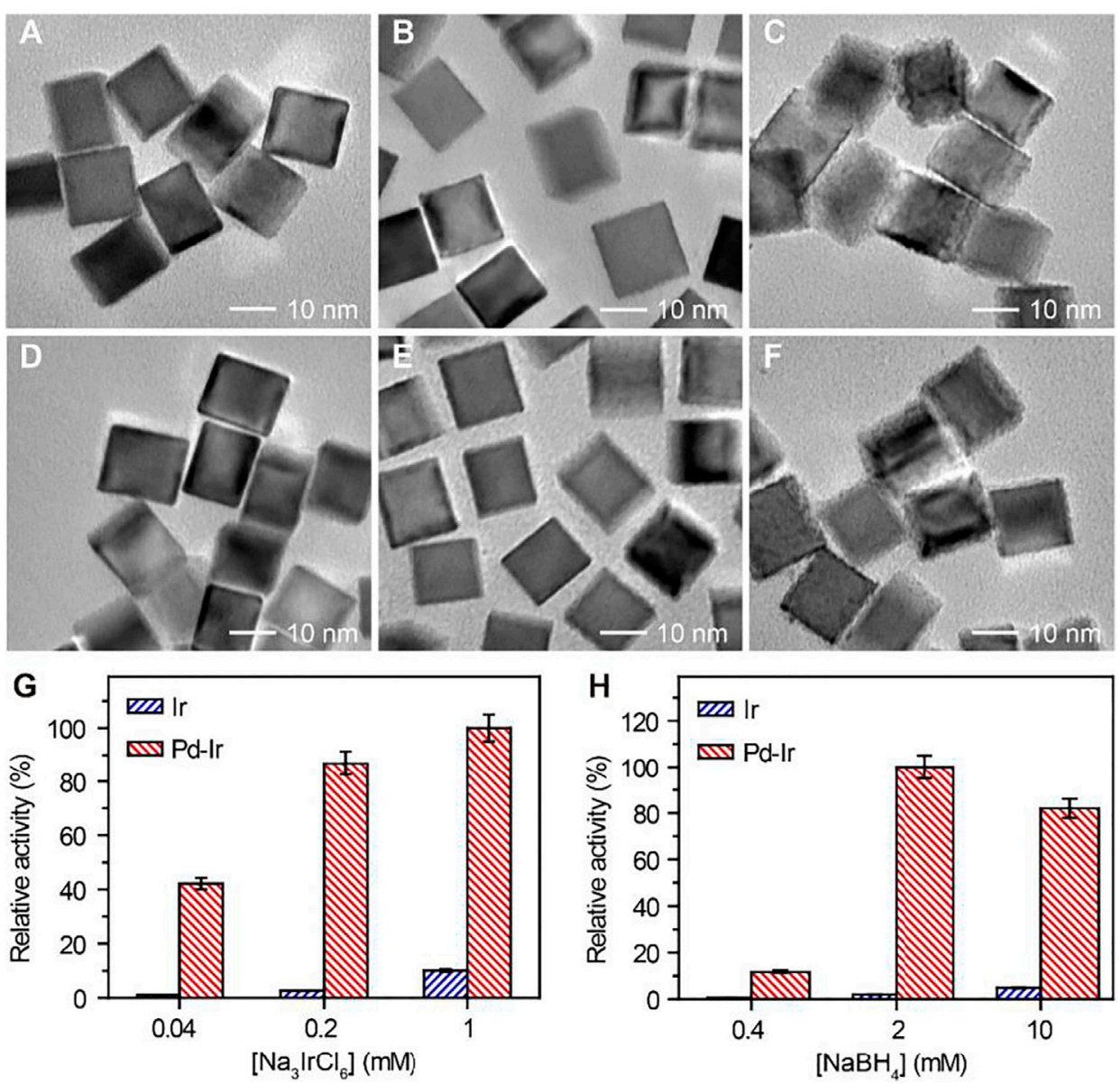

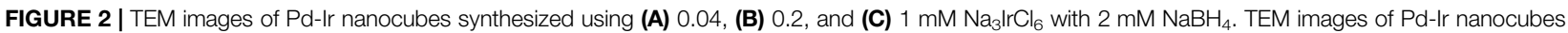
synthesized using (D) 0.4 , (E) 2, and (F) $10 \mathrm{mM} \mathrm{NaBH}_{4}$ with $0.2 \mathrm{mM} \mathrm{Na}_{3} \mathrm{ICl}_{6}$. Relative peroxidase mimicking activities of Pd-Ir nanocubes synthesized with different concentrations of (G) $\mathrm{Na}_{3} \mid \mathrm{rCl} 6$ and $\mathbf{( H )} \mathrm{NaBH}_{4}$.

about $0.8 \mathrm{~nm}$. Compared to Pd nanocubes, Pd-Ir nanocubes display a lighter brown appearance (Supplementary Figure S1H) with a decrease of absorbance around $450 \mathrm{~nm}$ (Supplementary Figure S1H). Overall, deposition of thin Ir layers on Pd-nanocubes is achieved by a simple and surfactant-free approach, breaking through the relatively high energy barrier in the synthesis of Pd-Ir nanocubes compared to homogeneous nucleation of Ir nanoparticles.

\section{Optimization of Ir Deposition on Pd Nanocubes}

To synthesize Pd-Ir nanocubes with high peroxidase activity while avoiding homogenous nucleation of Ir nanoparticles, we optimized the concentrations of $\mathrm{Na}_{3} \mathrm{IrCl}_{6}$ and $\mathrm{NaBH}_{4}$ for Ir deposition. Pd-Ir nanocubes from heterogeneous nucleation and Ir nanoparticles from homogeneous nucleation were separated by centrifugation and investigated for their peroxidase activities towards the oxidation of tetramethylbenzidine (TMB) with $\mathrm{H}_{2} \mathrm{O}_{2}$. As shown in Figure 2, amorphous $\mathrm{Ir}$ shells and significantly increased activity of Ir nanoparticles were observed when the concentrations of $\mathrm{Na}_{3} \mathrm{IrCl}_{6}$ were higher than $0.2 \mathrm{mM}$, or the concentration of $\mathrm{NaBH}_{4}$ over $2 \mathrm{mM}$. It indicates severe homogeneous nucleation happens at these conditions. To obtain Pd-Ir nanocubes with high catalytic activity and good batch consistency, the concentrations of $\mathrm{Na}_{3} \mathrm{IrCl}_{6}$ and $\mathrm{NaBH}_{4}$ were set to be 0.2 and $2 \mathrm{mM}$, respectively. This approach overcomes the difficulties in coating thin Ir layers on $\mathrm{Pd}$ nanocubes by controlling the reaction kinetic with $\mathrm{Na}_{3} \mathrm{IrCl}_{6}$ and $\mathrm{NaBH}_{4}$ concentrations. Moreover, the best activity was observed with the $\mathrm{Ir}$ deposition reaction time of $4 \mathrm{~h}$ (Supplementary Figure S2A) and with two additions of $\mathrm{NaBH}_{4}$ (Supplementary Figure S2B).

\section{Steady-State Kinetic Assays of Pd-Ir Nanocubes}

To investigate the peroxidase-like activity of Pd-Ir nanocubes, the steady-state kinetic constants for catalyzing the oxidation of TMB with $\mathrm{H}_{2} \mathrm{O}_{2}$ were determined using the Michaelis-Menten equation. The initial reaction velocities $(v)$ were plotted 

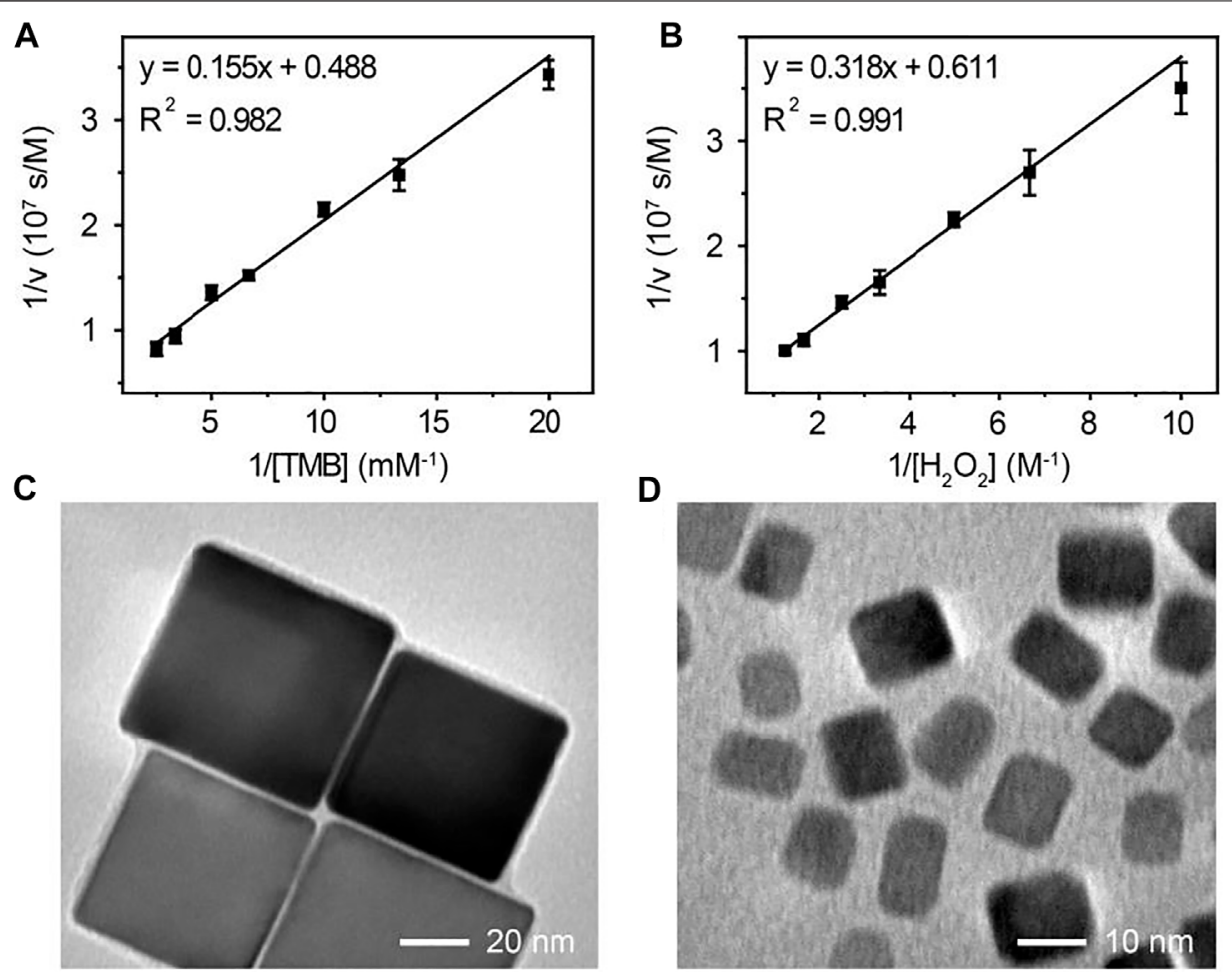

FIGURE 3 | The double-reciprocal plot of initial reaction velocity against (A) TMB or (B) $\mathrm{H}_{2} \mathrm{O}_{2}$ concentration, the reaction catalyzed by $18 \mathrm{~nm}$ Pd-lr nanocubes. The TEM images of (C) $51 \mathrm{~nm}$ and (D) $7 \mathrm{~nm}$ Pd-Ir nanocubes.

against the concentrations of TMB or $\mathrm{H}_{2} \mathrm{O}_{2}$ to obtain the Michaelis-Menten curves (Supplementary Figure S2C,D), which were then fitted to double-reciprocal plots (Figures 3A,B). The kinetic parameters of Pd-Ir nanocubes for TMB and $\mathrm{H}_{2} \mathrm{O}_{2}$ are listed in Supplementary Table S1. The catalytic rate constant $\left(k_{\text {cat }}\right)$ values of Pd-Ir nanocubes for TMB and $\mathrm{H}_{2} \mathrm{O}_{2}$ were higher compared to previously reported Pd-Ir nanocubes, which were about 3 orders of magnitude higher than that of HRP (Gao et al., 2007; Xia et al., 2015). The excellent peroxidase-like activity of Pd-Ir nanocubes is attributed to the intrinsic electronic shell structure of Ir, the electronic effect between Pd and Ir, and the absence of surfactants during synthesis.

To demonstrate the versatility of this method, Pd-Ir nanocubes with different sizes were synthesized. Pd nanocubes of 51 and $7 \mathrm{~nm}$ were synthesized according to the literature (Li and Li, 2021). As shown in Supplementary Figure S2E,F, both Pd nanocube species show well-defined cubic shapes, indicating that they are enclosed by (100) crystal faces. Thin Ir layers were then coated on the nanoparticle surface. Pd-Ir nanocubes still maintain the cubic crystal structure with a smooth surface (Figures 3C,D), demonstrating that this approach can also be applied for the preparation of 51 and $7 \mathrm{~nm}$ Pd-Ir nanocubes. The catalytic rate constants of Pd and Pd-Ir nanocubes with different sizes were compared (Figure 4A). The catalytic rate constant increases with particle sizes for both Pd and Pd-Ir nanocubes, likely due to the increased surface. Pd-Ir nanocubes show about two orders of magnitude higher peroxidase activity compared to corresponding Pd nanocubes.

\section{An Immunoassay for Prostate-Specific Antigen}

PSA, a serine protease for physiologically liquefying the seminal fluid (Lilja, 1985), has been used extensively as a cancer marker for initial diagnosis and monitoring response to treatment (Catalona et al., 1991; Barry, 2001; Wu et al., 2001; Lilja et al., 2008). Hence, PSA was chosen as the target to test the immunoassay performance of Pd-Ir nanocubes. Due to surfactant-free synthesis, Pd-Ir nanocubes can be conjugated with antibodies by physical adsorption, avoiding the timeconsuming and complex process of chemical conjugation (Xia et al., 2015). As shown in Figure 4B, Pd-Ir nanocubes were used as peroxidase-mimicking enzymes and functionalized with PSAspecific antibodies for the detection of PSA. In the presence of the target, detection antibody-conjugated Pd-Ir nanocubes are immobilized on capture antibody-coated microtiter plate through the specific antibody-antigen interaction. The immobilized Pd-Ir nanocubes catalyze the oxidation of colorless TMB to blue oxidized products $\left(\mathrm{TMB}^{+}\right)$, which turn yellow $\left(\mathrm{TMB}^{2+}\right)$ after termination with sulfuric acid. The concentration of PSA is determined by the UV-Vis absorbance at $450 \mathrm{~nm}$ by comparing with a standard curve. 

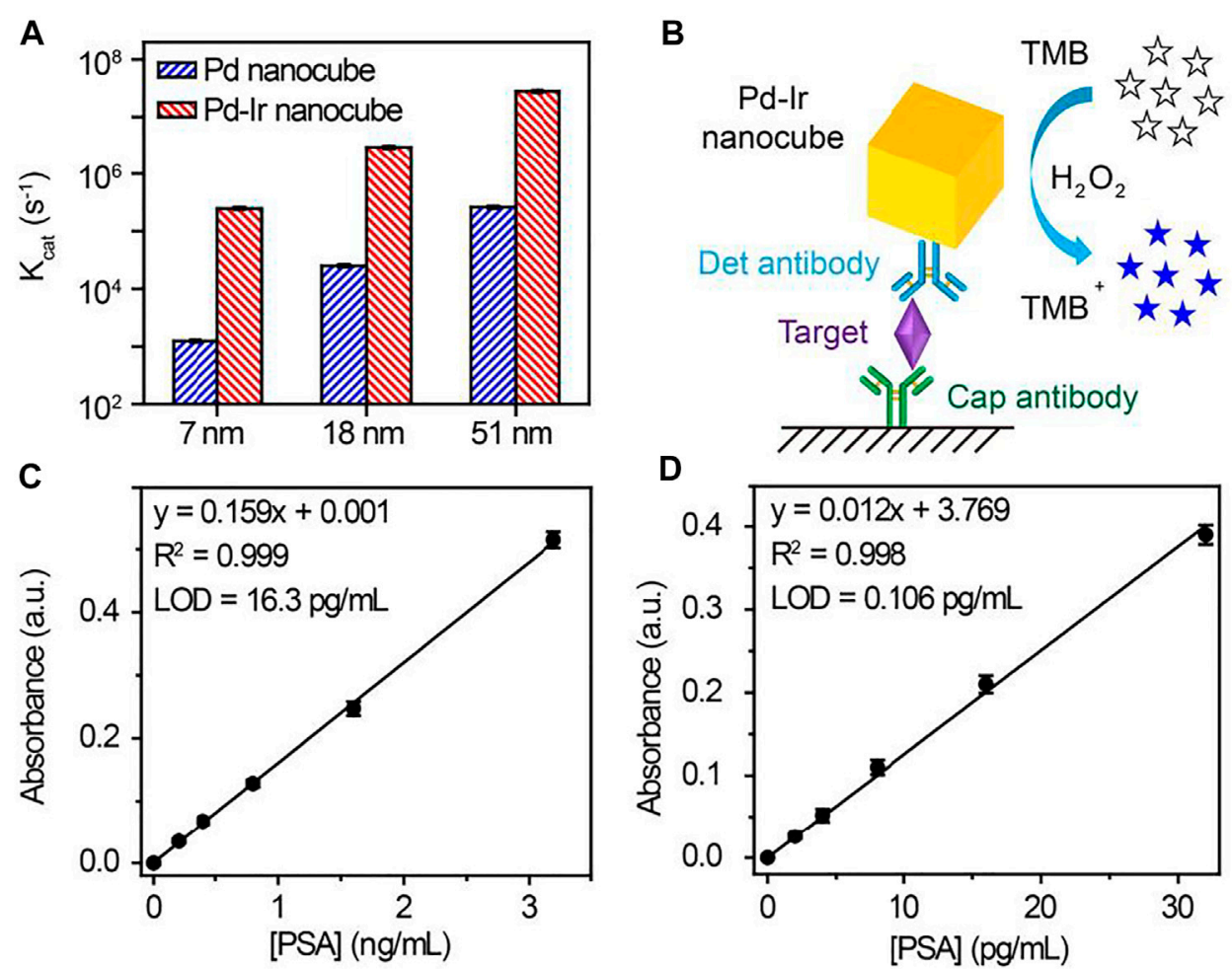

FIGURE 4 | (A) Catalytic rate constants of Pd and Pd-Ir nanocubes with different edge lengths. (B) Scheme of Pd-Ir nanocube-based ELISA for the detection of PSA. Signal responses of (C) HRP and (D) Pd-Ir nanocube-based ELISA for the detection of PSA. LOD (limit of detection) is defined as the concentration of targets corresponding to a signal that is three times the standard deviation higher than zero calibrators $(n=6)$.

We first optimize the conditions to functionalize Pd-Ir nanocubes with antibodies. Bovine serum albumin (BSA) is used to block Pd-Ir nanocubes and prevent non-specific adsorption. As shown in Supplementary Figure S3A, it needed $30 \mathrm{~min}$ to complete the attachment of antibodies on Pd-Ir nanocubes. $10 \% \mathrm{w} / \mathrm{v}$ BSA and $1 \mathrm{~h}$ incubation at room temperature were needed to block $\mathrm{Pd}-\mathrm{Ir}$ nanocubes (Supplementary Figure S3B,C). Thereafter, antibodyconjugated Pd-Ir nanocubes were used for the detection of PSA. As shown in Figures 4C,D, UV-Vis absorbance at $450 \mathrm{~nm}$ shows a linear relationship to the concentration of PSA for both HRP-based and Pd-Ir nanocube-based methods. The detection limits for HRP-based and Pd-Ir nanocube-based methods are $16.3 \mathrm{pg} / \mathrm{ml}$ and $0.106 \mathrm{pg} / \mathrm{ml}$, respectively. It demonstrates that Pd-Ir nanocube-based method has two orders of magnitude higher sensitivity than the HRP-based method.

\section{An Aptamer-Based Dual Fluorescent and Colorimetric Biosensor for C. Difficile RNase HII}

Pd-Ir nanocubes were also employed for the construction of dual fluorescent and colorimetric biosensors. Because Pd-Ir nanocubes are noble metal nanoparticles with six flat uniform crystal facets, it has great potential to improve the consistency of nucleic acid adsorption, which can be employed for the design of fluorescent biosensors. However, Pd-Ir nanocubes synthesized by the traditional method were capped by surfactant, which prevents the adsorption of nucleic acids. Due to surfactant-free synthesis, Pd-Ir nanocubes prepared in this report have great potential to adsorb nucleic acids and be used for fluorescent biosensors. ARH1t6, a DNA aptamer for C. difficile RNase HII (Li et al., 2021), was labelled with fluorescein to investigate the nucleic acid adsorption property of Pd-Ir nanocubes. As shown in Supplementary Figure S4A, FAM-ARH1t6 can be efficiently adsorbed to the surface of Pd-Ir nanocubes synthesized in this report compared to the ones synthesized according to literature (Xia et al., 2015). FAM-ARH1t6 was then combined with Pd-Ir nanocubes and used for the detection of $C$. difficile RNase HII. The detection scheme is shown in Figure 5A. In the absence of $C$. difficile RNase HII, FAM-ARH1t6 is adsorbed to the surface of Pd-Ir nanocubes, leading to efficient fluorescence quenching of FAM-ARH1t6 and catalytic activity inhibition of Pd-Ir nanocubes. However, in the presence of $C$. difficile RNase HII, FAM-ARH1t6 switches binding partners from Pd-Ir nanocubes to RNase HII, resulting in increases of both fluorescence intensity and peroxidase catalytic activity. As shown in Figures 5B,C, the fluorescence intensity is proportional to the concentrations of $C$. difficile RNase HII, which can be quantitatively detected from 0 to $4 \mathrm{nM}$ with a limit of detection (LOD) of $0.15 \mathrm{nM}$. Alternatively, $C$. difficile RNase HII can also be determined by the colorimetric 

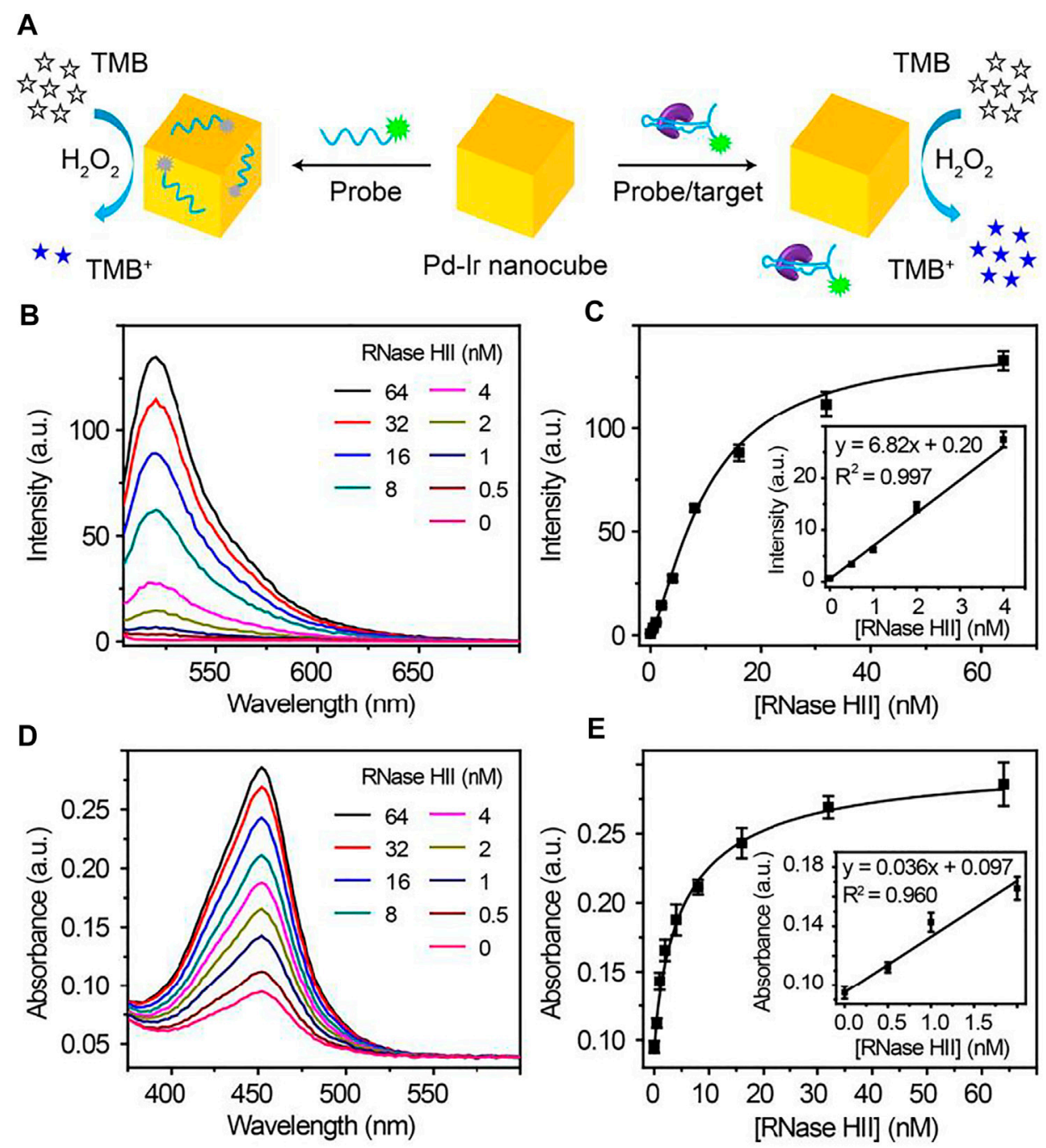

FIGURE 5 | (A) Schematic illustration of fluorescent and colorimetric detection of $C$. difficile RNase HII using Pd-Ir nanocubes and fluorescein-labelled aptamers. (B) Fluorescence curves and (C) plot (inset: linear response) of corresponding fluorescence intensity at $522 \mathrm{~nm}$ towards different concentrations of $C$. difficile RNase HII; $\mathrm{LOD}=0.15 \mathrm{nM}$. (D) Absorption curves and (E) plot (inset: linear response) of the corresponding absorbance at $450 \mathrm{~nm}$ towards different concentrations of $C$. difficile RNase HII; LOD $=0.29 \mathrm{nM}$. LOD (limit of detection) is defined as the concentration of targets corresponding to a signal that is three times the standard deviation higher than zero calibrators $(n=6)$.

method. A positive relationship is observed for the catalytic activity of $\mathrm{Pd}$-Ir nanocubes with target concentrations (Figure 5D). C. difficile RNase HII is quantitatively detected by the colorimetric approach in the $0-2 \mathrm{nM}$ range with a LOD of $0.29 \mathrm{nM}$ (Figure 5E). Moreover, this biosensor has good selectivity for the detection of $C$. difficile RNase HII compared to RNase HII from other bacteria (Supplementary Figure S4B). The results demonstrate that the fluorescent approach agrees well with the colorimetric approach. The combination of fluorescent and colorimetric approaches can improve detection accuracy.

To demonstrate the practical application of the Pd-Ir nanocube-based biosensors for real samples, we spiked different concentrations of $C$. difficile RNase HII in tap water (from our lab) in the range of $0.5-2 \mathrm{nM}$ and detected RNase HII by the fluorescent biosensors. As summarized in Supplementary Table S2, the recoveries for the four RNase HII spiked tap water samples were measured to be between 92.27 and $105.52 \%$. The coefficient of variation $(n=6)$ was below $10.13 \%$ for all samples. The results imply that the performance of Pd-Ir nanocube-based biosensors was not compromised by tap water, suggesting the feasibility of this biosensor for real sample detection.

\section{CONCLUSION}

In summary, a simple and surfactant-free method is developed here to synthesize Pd-Ir nanocubes with atomic Ir shells in an aqueous solution at room temperature. A long-standing issue 
with the coating of Ir on Pd nanocubes is addressed by simply adjusting the reaction kinetic with the concentrations of $\mathrm{Na}_{3} \mathrm{IrCl}_{6}$ and $\mathrm{NaBH}_{4}$. The Pd-Ir nanocubes have uniform particle size and excellent peroxidase-mimicking activity, due to the intrinsic electronic structure of Ir, electronic effect between Pd and Ir, and clean surface. Pd nanocubes with different sizes can be coated with atomic Ir layers using this approach. Due to its surfactantfree synthesis, Pd-Ir nanocubes can be conjugated with antibodies by physical adsorption for immunoassay, avoiding the tedious and complex chemical conjugation processes. Pd-Ir nanocubes can also be combined with fluorescent aptamers for the detection of $C$. difficile RNase HII by both fluorescence and colorimetric reporting. The method presented here makes a significant step forward towards the practical application of Pd-Ir nanocubes for biosensing.

\section{DATA AVAILABILITY STATEMENT}

The original contributions presented in the study are included in the article/Supplementary Material, further inquiries can be directed to the corresponding author.

\section{REFERENCES}

André, R., Natálio, F., Humanes, M., Leppin, J., Heinze, K., Wever, R., et al. (2011). V2O5 Nanowires with an Intrinsic Peroxidase-like Activity. Adv. Funct. Mater. 21, 501-509. doi:10.1002/adfm.201001302

Anker, J. N., Hall, W. P., Lyandres, O., Shah, N. C., Zhao, J., and Van Duyne, R. P. (2008). Biosensing with Plasmonic Nanosensors. Nat. Mater 7, 442-453. doi:10.1038/nmat2162

Barry, M. J. (2001). Prostate-Specific-Antigen Testing for Early Diagnosis of Prostate Cancer. N. Engl. J. Med. 344, 1373-1377. doi:10.1016/S00904295(99)00271-X10.1056/nejm200105033441806

Burda, C., Chen, X., Narayanan, R., and El-Sayed, M. A. (2005). Chemistry and Properties of Nanocrystals of Different Shapes. Chem. Rev. 105, 1025-1102. doi:10.1021/cr030063a

Catalona, W. J., Smith, D. S., Ratliff, T. L., Dodds, K. M., Coplen, D. E., Yuan, J. J. J., et al. (1991). Measurement of Prostate-specific Antigen in Serum as a Screening Test for Prostate Cancer. N. Engl. J. Med. 324, 1156-1161. doi:10.1056/ nejm 199104253241702

Chen, W., Chen, J., Liu, A. L., Wang, L. M., Li, G. W., and Lin, X. H. (2011). Peroxidase-Like Activity of Cupric Oxide Nanoparticle. ChemCatChem 3, 1151-1154. doi:10.1002/cctc.201100064

Gao, L., Zhuang, J., Nie, L., Zhang, J., Zhang, Y., Gu, N., et al. (2007). Intrinsic Peroxidase-like Activity of Ferromagnetic Nanoparticles. Nat. Nanotech 2, 577-583. doi:10.1038/nnano.2007.260

He, S., Song, B., Li, D., Zhu, C., Qi, W., Wen, Y., et al. (2010a). A Graphene Nanoprobe for Rapid, Sensitive, and Multicolor Fluorescent Dna Analysis. Adv. Funct. Mater. 20, 453-459. doi:10.1002/adfm.200901639

He, W., Liu, Y., Yuan, J., Yin, J.-J., Wu, X., Hu, X., et al. (2011). Au@Pt Nanostructures as Oxidase and Peroxidase Mimetics for Use in Immunoassays. Biomaterials 32, 1139-1147. doi:10.1016/ j.biomaterials.2010.09.040

He, W., Wu, X., Liu, J., Hu, X., Zhang, K., Hou, S., et al. (2010b). Design of AgM Bimetallic Alloy Nanostructures $(\mathrm{M}=\mathrm{Au}, \mathrm{Pd}, \mathrm{Pt})$ with Tunable Morphology and Peroxidase-like Activity. Chem. Mater. 22, 2988-2994. doi:10.1021/ $\mathrm{cm} 100393 \mathrm{v}$

Jia, J., Wang, B., Wu, A., Cheng, G., Li, Z., and Dong, S. (2002). A Method to Construct a Third-Generation Horseradish Peroxidase Biosensor: SelfAssembling Gold Nanoparticles to Three-Dimensional Sol-Gel Network. Anal. Chem. 74, 2217-2223. doi:10.1021/ac011116w

\section{AUTHOR CONTRIBUTIONS}

JL: investigation, methodology and writing. YL: review and editing.

\section{FUNDING}

This work was supported by funding from Natural Sciences and Engineering Research Council of Canada (NSERC) via a Discovery Grant to YL (Grant Number RGPIN-2020-06401). The electron microscopy work was carried out at the Canadian Centre for Electron microscopy supported by CFI, NSERC, and McMaster University. Special appreciation goes to Jimmy Gu for commenting on the manuscript.

\section{SUPPLEMENTARY MATERIAL}

The Supplementary Material for this article can be found online at: https://www.frontiersin.org/articles/10.3389/fchem.2021.775220/ full\#supplementary-material

Jv, Y., Li, B., and Cao, R. (2010). Positively-charged Gold Nanoparticles as Peroxidiase Mimic and Their Application in Hydrogen Peroxide and Glucose Detection. Chem. Commun. 46, 8017-8019. doi:10.1039/c0cc02698k

Kaufman, C. M., and Sen, B. (1985). Hydrogen Generation by Hydrolysis of Sodium Tetrahydroborate: Effects of Acids and Transition Metals and Their Salts. J. Chem. Soc. Dalton Trans., 307-313. doi:10.1039/DT9850000307

Li, J., Gu, J., Zhang, H., Liu, R., Zhang, W., Mohammed-Elsabagh, M., et al. (2021). A Highly Specific DNA Aptamer for RNase H2 from clostridium Difficile. ACS Appl. Mater. Inter. 13, 9464-9471. doi:10.1021/acsami.0c20277

Li, J., and Li, Y. (2021). One-pot High-Yield Synthesis of Pd Nanocubes for Pd-Ir Nanocube-Based Immunoassay of Nucleocapsid Protein from SARS-CoV-2. Anal. Bioanal. Chem. 413, 4635-4644. doi:10.1007/s00216-021-03265-z

Lilja, H. (1985). A Kallikrein-like Serine Protease in Prostatic Fluid Cleaves the Predominant Seminal Vesicle Protein. J. Clin. Invest. 76, 1899-1903. doi:10.1172/JCI112185

Lilja, H., Ulmert, D., and Vickers, A. J. (2008). Prostate-specific Antigen and Prostate Cancer: Prediction, Detection and Monitoring. Nat. Rev. Cancer 8, 268-278. doi:10.1038/nrc2351

Lim, B., Jiang, M., Tao, J., Camargo, P. H. C., Zhu, Y., and Xia, Y. (2009). Shapecontrolled Synthesis of Pd Nanocrystals in Aqueous Solutions. Adv. Funct. Mater. 19, 189-200. doi:10.1002/adfm.200801439

Lin, Y., Ren, J., and Qu, X. (2014). Catalytically Active Nanomaterials: A Promising Candidate for Artificial Enzymes. Acc. Chem. Res. 47, 1097-1105. doi:10.1021/ $\operatorname{ar} 400250 \mathrm{z}$

Link, S., and El-Sayed, M. A. (1999). Spectral Properties and Relaxation Dynamics of Surface Plasmon Electronic Oscillations in Gold and Silver Nanodots and Nanorods. J. Phys. Chem. B 103, 8410-8426. doi:10.1021/jp9917648

Liu, M., Zheng, Y., Xie, S., Li, N., Lu, N., Wang, J., et al. (2013). Facile Synthesis of Pd-Ir Bimetallic Octapods and Nanocages through Galvanic Replacement and Co-reduction, and Their Use for Hydrazine Decomposition. Phys. Chem. Chem. Phys. 15, 11822-11829. doi:10.1039/c3cp51950c

Loh, K. P., Bao, Q., Eda, G., and Chhowalla, M. (2010). Graphene Oxide as a Chemically Tunable Platform for Optical Applications. Nat. Chem 2, 1015-1024. doi:10.1038/nchem.907

Lu, C.-H., Yang, H.-H., Zhu, C.-L., Chen, X., and Chen, G.-N. (2009). A Graphene Platform for Sensing Biomolecules. Angew. Chem. Int. Ed. 48, 4785-4787. doi:10.1002/anie.200901479

Migowski, P., Zanchet, D., Machado, G., Gelesky, M. A., Teixeira, S. R., and Dupont, J. (2010). Nanostructures in Ionic Liquids: Correlation of Iridium Nanoparticles' Size and Shape with Imidazolium Salts' Structural Organization 
and Catalytic Properties. Phys. Chem. Chem. Phys. 12, 6826-6833. doi:10.1039/ b925834e

Mu, J., Wang, Y., Zhao, M., and Zhang, L. (2012). Intrinsic Peroxidase-like Activity and Catalase-like Activity of $\mathrm{Co} 3 \mathrm{O} 4$ Nanoparticles. Chem. Commun. 48, 2540-2542. doi:10.1039/c2cc17013b

Muir, S. S., and Yao, X. (2011). Progress in Sodium Borohydride as a Hydrogen Storage Material: Development of Hydrolysis Catalysts and Reaction Systems. Int. J. Hydrogen Energ. 36, 5983-5997. doi:10.1016/j.ijhydene.2011.02.032

Peng, Y., Huang, Y., Zhu, Y., Chen, B., Wang, L., Lai, Z., et al. (2017). Ultrathin Two-Dimensional Covalent Organic Framework Nanosheets: Preparation and Application in Highly Sensitive and Selective DNA Detection. J. Am. Chem. Soc. 139, 8698-8704. doi:10.1021/jacs.7b04096

Retnamma, R., Novais, A. Q., and Rangel, C. M. (2011). Kinetics of Hydrolysis of Sodium Borohydride for Hydrogen Production in Fuel Cell Applications: a Review. Int. J. Hydrogen Energ. 36, 9772-9790. doi:10.1016/ j.ijhydene.2011.04.223

Saha, K., Agasti, S. S., Kim, C., Li, X., and Rotello, V. M. (2012). Gold Nanoparticles in Chemical and Biological Sensing. Chem. Rev. 112, 2739-2779. doi:10.1021/ cr2001178

Song, Y., Qu, K., Zhao, C., Ren, J., and Qu, X. (2010a). Graphene Oxide: Intrinsic Peroxidase Catalytic Activity and its Application to Glucose Detection. Adv. Mater. 22, 2206-2210. doi:10.1002/adma.200903783

Song, Y., Wang, X., Zhao, C., Qu, K., Ren, J., and Qu, X. (2010b). Label-Free Colorimetric Detection of Single Nucleotide Polymorphism by Using SingleWalled Carbon Nanotube Intrinsic Peroxidase-like Activity. Chem. Eur. J. 16, 3617-3621. doi:10.1002/chem.200902643

Stowell, C. A., and Korgel, B. A. (2005). Iridium Nanocrystal Synthesis and Surface Coating-dependent Catalytic Activity. Nano Lett. 5, 1203-1207. doi:10.1021/ nl050648f

Tan, C., Yu, P., Hu, Y., Chen, J., Huang, Y., Cai, Y., et al. (2015). High-yield Exfoliation of Ultrathin Two-Dimensional Ternary Chalcogenide Nanosheets for Highly Sensitive and Selective Fluorescence DNA Sensors. J. Am. Chem. Soc. 137, 10430-10436. doi:10.1021/jacs.5b06982

Wang, Y., Li, Z., Hu, D., Lin, C.-T., Li, J., and Lin, Y. (2010). Aptamer/graphene Oxide Nanocomplex for In Situ Molecular Probing in Living Cells. J. Am. Chem. Soc. 132, 9274-9276. doi:10.1021/ja103169v

Wang, Y., Li, Z., Wang, J., Li, J., and Lin, Y. (2011). Graphene and Graphene Oxide: Biofunctionalization and Applications in Biotechnology. Trends Biotechnol. 29, 205-212. doi:10.1016/j.tibtech.2011.01.008

Wei, H., and Wang, E. (2008). Fe3O4 Magnetic Nanoparticles as Peroxidase Mimetics and Their Applications in $\mathrm{H} 2 \mathrm{O} 2$ and Glucose Detection. Anal. Chem. 80, 2250-2254. doi:10.1021/ac702203f

Wei, H., and Wang, E. (2013). Nanomaterials with Enzyme-like Characteristics (Nanozymes): Next-Generation Artificial Enzymes. Chem. Soc. Rev. 42, 6060-6093. doi:10.1039/c3cs35486e

Willets, K. A., and Van Duyne, R. P. (2007). Localized Surface Plasmon Resonance Spectroscopy and Sensing. Annu. Rev. Phys. Chem. 58, 267-297. doi:10.1146/ annurev.physchem.58.032806.104607
Wu, G., Datar, R. H., Hansen, K. M., Thundat, T., Cote, R. J., and Majumdar, A. (2001). Bioassay of Prostate-specific Antigen (PSA) Using Microcantilevers. Nat. Biotechnol. 19, 856-860. doi:10.1038/nbt0901-856

Wu, J., Wang, X., Wang, Q., Lou, Z., Li, S., Zhu, Y., et al. (2019). Nanomaterials with Enzyme-like Characteristics (Nanozymes): Next-Generation Artificial Enzymes (II). Chem. Soc. Rev. 48, 1004-1076. doi:10.1039/c8cs00457a

Xia, X., Figueroa-Cosme, L., Tao, J., Peng, H.-C., Niu, G., Zhu, Y., et al. (2014). Facile Synthesis of Iridium Nanocrystals with Well-Controlled Facets Using Seed-Mediated Growth. J. Am. Chem. Soc. 136, 10878-10881. doi:10.1021/ ja505716v

Xia, X., Zhang, J., Lu, N., Kim, M. J., Ghale, K., Xu, Y., et al. (2015). Pd-Ir Core-Shell Nanocubes: A Type of Highly Efficient and Versatile Peroxidase Mimic. ACS Nano 9, 9994-10004. doi:10.1021/acsnano.5b03525

Ye, H., Yang, K., Tao, J., Liu, Y., Zhang, Q., Habibi, S., et al. (2017). An Enzyme-free Signal Amplification Technique for Ultrasensitive Colorimetric Assay of Disease Biomarkers. ACS Nano 11, 2052-2059. doi:10.1021/acsnano.6b08232

Zanata, C. R., Martins, C. A., Teixeira-Neto, É., Giz, M. J., and Camara, G. A. (2019). Two-step Synthesis of Ir-Decorated Pd Nanocubes and Their Impact on the Glycerol Electrooxidation. J. Catal. 377, 358-366. doi:10.1016/ j.jcat.2019.07.042

Zhu, C., Zeng, Z., Li, H., Li, F., Fan, C., and Zhang, H. (2013). Single-Layer MoS2Based Nanoprobes for Homogeneous Detection of Biomolecules. J. Am. Chem. Soc. 135, 5998-6001. doi:10.1021/ja4019572

Zhu, J., Chen, Z., Xie, M., Lyu, Z., Chi, M., Mavrikakis, M., et al. (2019a). IridiumBased Cubic Nanocages with 1.1-nm-Thick Walls: A Highly Efficient and Durable Electrocatalyst for Water Oxidation in an Acidic Medium. Angew. Chem. Int. Ed. 58, 7244-7248. doi:10.1002/anie.201901732

Zhu, J., Lyu, Z., Chen, Z., Xie, M., Chi, M., Jin, W., et al. (2019b). Facile Synthesis and Characterization of Pd@IrnL (N = 1-4) Core-Shell Nanocubes for Highly Efficient Oxygen Evolution in Acidic Media. Chem. Mater. 31, 5867-5875. doi:10.1021/acs.chemmater.9b02011

Conflict of Interest: The authors declare that the research was conducted in the absence of any commercial or financial relationships that could be construed as a potential conflict of interest.

Publisher's Note: All claims expressed in this article are solely those of the authors and do not necessarily represent those of their affiliated organizations, or those of the publisher, the editors, and the reviewers. Any product that may be evaluated in this article, or claim that may be made by its manufacturer, is not guaranteed or endorsed by the publisher.

Copyright $\odot 2021 \mathrm{Li}$ and Li. This is an open-access article distributed under the terms of the Creative Commons Attribution License (CC BY). The use, distribution or reproduction in other forums is permitted, provided the original author(s) and the copyright owner(s) are credited and that the original publication in this journal is cited, in accordance with accepted academic practice. No use, distribution or reproduction is permitted which does not comply with these terms. 